\title{
The development of tumours under a ketogenic diet in association with the novel tumour marker TKTL1: A case series in general practice
}

\author{
NATALIE JANSEN ${ }^{1,2}$ and HARALD WALACH ${ }^{2}$ \\ ${ }^{1}$ Dr Natalie Jansen Private Practice, Heilbronn, Baden-Württemberg, D-74074 Heilbronn; \\ ${ }^{2}$ Institute of Transcultural Health Studies, European University Viadrina, D-15207 Frankfurt (Oder), Germany
}

Received November 17, 2014; Accepted July 21, 2015

DOI: $10.3892 / \mathrm{ol} .2015 .3923$

\begin{abstract}
Since the initial observations by Warburg in 1924, it has become clear in recent years that tumour cells require a high level of glucose to proliferate. Therefore, a ketogenic diet that provides the body with energy mainly through fat and proteins, but contains a reduced amount of carbohydrates, has become a dietary option for supporting tumour treatment and has exhibited promising results. In the present study, the first case series of such a treatment in general practice is presented, in which 78 patients with tumours were treated within a time window of 10 months. The patients were monitored regarding their levels of transketolase-like-1 (TKTL1), a novel tumour marker associated with aerobic glycolysis of tumour cells, and the patients' degree of adherence to a ketogenic diet. Tumour progression was documented according to oncologists' reports. Tumour status was correlated with TKTL1 expression (Kruskal-Wallis test, $\mathrm{P}<0.0001)$, indicating that more progressed and aggressive tumours may require a higher level of aerobic glycolysis. In palliative patients, a clear trend was observed in patients who adhered strictly to a ketogenic diet, with one patient experiencing a stagnation in tumour progression and others an improvement in their condition. The adoption of a ketogenic diet was also observed to affect the levels of TKTL1 in those patients. In conclusion, the results from the present case series in general practice suggest that it may be beneficial to advise tumour patients to adopt a ketogenic diet, and that those who adhere to it may have positive results from this type of diet. Thus, the use of a ketogenic diet as a complementary treatment to tumour therapy must be further studied in rigorously controlled trials.
\end{abstract}

Correspondence to: Professor Harald Walach, Institute of Transcultural Health Studies, European University Viadrina, 59 Große Scharmstr, D-15207 Frankfurt (Oder), Germany

E-mail:walach@europa-uni.de

Key words: cancer, diet, aerobic glycolysis, TKTL1, ketogenic diet, case series

\section{Introduction}

Despite the 'war on cancer' propagated by the World Health Organisation (WHO) and the US government in the 1980s, cancer remains a serious threat to health worldwide, particularly in Western countries (1). While the number of cases of certain types of cancer, such as gastrointestinal cancer, have declined, others such as breast cancer are on the rise. As a result, cancer is the second most common cause of premature mortality in Western countries and the third most common worldwide (2). In 2014, the WHO predicted that the incidence of cancer will approximately duplicate over the proceeding 2 decades. It also estimated that $\geq 30 \%$ of those cases are preventable through public health strategies to combat the leading causes, the most notable of which are a high body mass index (BMI), a diet lacking natural antioxidants and the abuse of alcohol and tobacco (3). This brings dietary interventions into focus for the prevention and possible treatment of cancer $(4,5)$. Although extensive studies and resources have been invested into cancer research and diagnosis, the survival rates have barely altered for certain types of cancer (6-9).

In recent years, attention was drawn to the fact that diet-associated factors may be involved in the development and progression of cancer. The Harvard Alumni Health Study demonstrated an association between low BMI at early adulthood and late mortality from cancer, with an increased risk of $11 \%$ for each standard deviation unit of increased BMI at 18 years of age. This association was valid regardless of the tumour site (10). The European Prospective Investigation into Cancer and Nutrition Study observed a $36 \%$ reduction in the risk of cancer mortality for people living a healthy lifestyle, which included exercise, maintaining a healthy weight, no smoking and following a healthy diet (11). This reduction was higher for specific types of cancer (12). According to Donaldson (13) and Bao et al (14), the consumption of foods rich in antioxidants and healthy combinations of fats and carbohydrates such as nuts may protect against cancer mortality.

Therefore, it is not surprising that in recent years, the potential of a ketogenic diet as an adjunct to standard cancer care has raised interest among researchers. In a ketogenic diet, the energy requirements of the organism are met by a diet rich in proteins and fats with comparatively few carbohydrates, 
normally in a ratio of 3 parts fats and proteins to $\leq 1$ part carbohydrates $(3,15)$. This is a nutritional approach that mimics physiological fasting, which may be beneficial in the treatment of cancer, if it is moderate and not prolonged, as suggested by animal models and clinical experience (16). During fasting, the body converts triglycerides from fat stores into ketone bodies, including acetoacetate, acetone and $\beta$-hydroxybutyrate, which can be used as an energy supply by the majority of cells, including muscle cells and neurons (15). For those cells that require sugar, gluconeogenesis can generate sugar de novo from the glycerol that is produced during lipolysis, and thus, the levels of sugar in blood stay within the normal range during ketosis $(4,15)$. It is noteworthy that a therapeutic ketogenic diet is physiologically close to the 'paleolithic diet' that is currently discussed as potentially beneficial for weight loss (17-19). A ketogenic diet has been demonstrated to be therapeutically useful for the treatment of epilepsy (15) and cardiovascular diseases (4).

In 1924, Otto Warburg discovered that cancer cells can generate energy from sugar without oxidation, regardless of the presence of oxygen, and named this phenomenon 'aerobic glycolysis' $(20,21)$. Glycolysis is an inefficient way to generate energy, since it converts 1 glucose molecule into 2 molecules of pyruvate, and the energy released is then used to produce 2 molecules of ATP, whereas through oxidation the yield of 1 molecule of glucose is $~ 38$ molecules of ATP $(22,23)$. Glycolysis is present in primitive organisms such as bacteria that can exist without oxygen, and is possibly one of the oldest mechanisms providing organisms with energy (24). Glycolysis is used by human cells and neurons (25) under high stress or anaerobic conditions (26). Cells normally oxidise sugar because it provides them with more energy than glycolysis does, but, in contrast to glycolysis, oxidation produces free radicals that must be buffered, since they may damage cells and cellular structures. This is the rationale behind numerous cancer treatments that increase oxidative stress (26). Warburg hypothesized that cancer cells switch to glycolysis, as their mitochondria are dysfunctional. However, this was demonstrated to be wrong, and in consequence, Warburg's hypothesis was rejected (27). A more modern and enlightened view proposes that cancer cells switch to glycolysis, despite oxygen being present, as they can thereby escape the apoptotic mechanisms that are induced by free radicals $(23,26,28)$, and it facilitates the incorporation of other components necessary for cell growth (22). However, this process creates a heavy demand on glucose supply, since the growth of cancer cells exclusively occurs if there is enough glucose provision. Furthermore, cancer cells appear to be able to use ketone bodies for energy production under certain restricted conditions, and solely if enough oxygen is present, which is not the case at those sites where the tumour grows rapidly $(29,30)$. Thus, depriving cancer cells of glucose may reinstate apoptosis in cancer cells (31). Furthermore, high fasting insulin levels that lead to insulin resistance has been associated with a 2.4-fold risk of developing breast cancer in post-menopausal women (32). Therefore, the use of a ketogenic diet as a supportive strategy for patients with tumours is a rational and empirically supported approach. This type of diet provides daily $\sim 1 \mathrm{~g}$ carbohydrates/kg body weight $(28,33)$, combined with unsaturated fatty acids, particularly $\Omega-3$, short- and medium-chain fatty acids. A good supply of plant-derived antioxidants, such as polyphenols, and a lactate drink to buffer acidity are also important. Brochures and books regarding the use of ketogenic diet for patients with cancer have been available in Germany for several years $(34,35)$.

The transketolase-like-1 (TKTL1) gene was identified as a mutation of the transketolase gene. The TKTL1 enzyme and its associated proteins have been associated with switching the cell into aerobic glycolysis as a secondary pathway to generate energy $(36,37)$. TKTL1 seems to boost the glucose metabolism of cancer cells (38), and its activity has been associated with anaerobic glycolysis in cancer cells and with low survival rates (39). By contrast, the inhibition of TKTL1 expression has been observed to be associated with reduced proliferation in a hepatoma cell model (40). Previous studies have demonstrated that TKTL1 is an independent oncogenic marker, possibly associated with an enhanced glucose metabolism, and that increased serum levels of TKTL1 are associated with the recurrence of cancer in patients with oral squamous cell carcinoma, breast cancer and prostate cancer (39,41-43). TKTL1 can be reliably measured using a novel immunological assay, termed the epitope detection in monocyte (EDIM) test, with $94 \%$ sensitivity and $82 \%$ specificity. The EDIM test presents a positive predictive value of $87 \%$, and correlates $90 \%$ with the current standard procedure, a positron emission tomography (PET) scan to measure radioactive glucose incorporation into cancer cells (44). In one clinical case, it was observed that the levels of TKTL1 were increased in a patient following resection of colon cancer, and prior to the appearance of clinically visible metastases, thus demonstrating the practical use and sensitivity of TKTL1 as a marker (45). Although negative findings have also been reported (46), collectively, these observations indicate that TKTL1 may act as a genetic switch, enabling cancer cells to perform aerobic glycolysis in order to meet their increased demand on glucose supply required for proliferation. In addition, Apo10 overexpression has been detected in different types of tumors. It is assoctiated with the inhibition of of apoptosis and is indicative of abnormal cell proliferation. As of 2010, it has also been possible to measure Apo10 levels using the EDIM blood test (47).

It is plausible to assume that a ketogenic diet operates on this basis, and therefore bears certain promise. Previous pilot and feasibility trials have established the potential of a ketogenic diet as supportive treatment against cancer. In a small pilot study with 16 patients with cancer, the ketogenic diet was accepted well by 9 patients, and 5 of them completed the study. In those 5 patients who were able to adhere to the diet, the disease was stable without further progress, and their emotional function and sleep improved (33). In a small feasibility study in 10 patients with advanced cancer, 4 patients were unable to adhere to the diet, and 5 of those that were able to follow the 4-week diet reached physiological ketosis and experienced a remission or a retardation of progression of the disease. In these patients, the level of ketosis was significantly associated with reduced insulin levels (48). In another study, 20 patients with recurrent glioblastoma were administered a ketogenic diet (50). Those who reached ketosis experienced slower tumour progression. Overall, the diet was well received, although the blood sugar levels did not reduce as markedly as was anticipated, which was assumed by the authors to be due 
to steroid treatment. Taken together, these results are encouraging, and require further confirmation in larger randomised studies.

However, general practitioners, who have the task of caring for patients once standard tumour treatment has been provided, are frequently challenged by patients who want to contribute to the maintenance of a tumour-free status or avoid recurrence or metastatic development. Often, these patients possess a certain notion of the ketogenic diet, instilled by popular literature. In the present study, the effects of a ketogenic diet in a prospective cohort of tumour patients in general practice was studied in order to evaluate whether the levels of TKTL1 are associated with tumour status and progression, and are affected by a ketogenic diet, and whether adherence to a ketogenic diet is associated with a better prognosis in patients with cancer.

\section{Materials and methods}

Patient cohort. The present study was designed as a systematic, prospective cohort study in general practice. A total of 78 patients that had been diagnosed with any type of cancer since January 2001 were eligible for the study. The patients were examined in the private practice clinic of Heilbronn (Baden-Württemberg, Germany) between November 2010 and December 2011. The patients were screened and included in the present observational study if they had been diagnosed with any type of tumour, or had experienced the recurrence of a tumour or metastasising disease during the 10 years previous to the initiation of the study. There were no exclusion criteria. For all patients, except 1 that declined testing, the levels of TKTL1 were measured using the EDIM test, according to previously published procedures $(44,45)$, and controlled every $\sim 3$ months. The patients were followed-up in general practice until the end of 2011.

Treatment and patient follow-up. For the corresponding analyses, $3 \mathrm{ml}$ venous blood was extracted and preserved in a vial containing EDTA. Blood samples were stored at room temperature and sent by courier to the laboratory (Biovis Diagnostik MVZ GmbH, Limburg an der Lahn, Germany), where they were processed within $24 \mathrm{~h}$. The recommended precautions were followed, including not testing during immunosuppressive therapy with antibiotics or cortisone, or during the 4 weeks subsequent to surgery, chemotherapy or radiation therapy. Patients were instructed regarding the potential benefit of a ketogenic diet, particularly those patients whose TKTL1 levels were above the standard laboratory norms. For ease of implementation, patients were informed about the company Tavarlin (Frankfurt, Germany), which specialises in producing and mailing food that meets the requirements of a ketogenic diet, including oil, fat, snacks, bread, protein and energy drinks (35). Every 3 months, the patients were questioned regarding their eating habits in a clinical interview, and classified accordingly into 3 different groups (no ketogenic diet; partially ketogenic diet; or strict ketogenic diet), following a conservative criteria of classification: If the patients adhered fully to a ketogenic diet, they were classified as fully ketogenic. If they adhered partially to a ketogenic diet, or switched during the observational period from no ketogenic diet to ketogenic diet or vice versa, they were classified as partially ketogenic. All other patients were classified as not ketogenic.

Patients were also distributed, using their oncologists' reports, into the following categories: Preoperative; curative; palliative with improvement; palliative with stable disease; or palliative with progressing disease. As certain categories contained very few patients, the preoperative and curative categories were collapsed into curative, palliative with improvement and palliative with stable disease were collapsed into palliative, and palliative with progressing disease was renamed endstage.

The variation in TKTL1 scores was calculated from the end of the observation period to baseline. Thus, negative scores corresponded to a reduction in TKTL1 values, while positive scores represented an increase over time. Patients were then grouped according to the following criteria: Those patients that did not initiate a ketogenic diet or returned to their ordinary diet; those that adhered partially to a ketogenic diet; and those that completely embraced a ketogenic diet. Patients were categorised according to their variation in tumour status from the beginning to the end of the observation period, by subtracting the initial category from the last one available. Thus, a negative score indicated an improvement in tumour status over time (for example, from palliative with progress to palliative stable, or from palliative to curative), while a positive score corresponded to a deterioration of the disease. All the categories of deterioration were collapsed into 1 category to facilitate the analysis and representation of the results.

Statistical analysis. Robust non-parametric, descriptive statistics and graphical analysis were used to display the data. Yates' correction for continuity was applied to tables with small cells. Statistical analyses were performed using Statistica software, version 8 (StatSoft, Ltd., Bedford, UK). Due to the absence of systematic knowledge from previous studies, all data, tests and significances in the present study must be interpreted with caution, and considered as orienting and exploratory. $\mathrm{P}<0.05$ was considered to indicate a statistically significant difference.

\section{Results}

Correlation between TKTL1 values, patient adherence to ketogenic diet and disease progression. A total of 78 patients with different types of tumours were included in the present study. The characteristics of the patients, including age, gender, BMI, smoking habits, maximum values of TKTL1 and Apo10, time since diagnosis, tumour stage, type of cancer and adherence to a ketogenic diet, are presented in Tables I and II. Maximum values of TKTL1 and Apo10 were determined by measuring every 3 months, and the maximum value was selected as the cancer is considered to be most aggressive at this point.

Of the 78 patients included in the study, 18 presented with breast cancer, 16 with prostate cancer, 9 with colon cancer, 2 with melanoma, 5 with lung cancer, 5 with otolaryngological cancer and 23 with other types of cancer. In these patients, $56(72 \%)$ cases were classified as being at a curative stage, $15(19 \%)$ at a palliative stage, and $7(9 \%)$ at an endstage. The correlations between the maximum values of TKTL1 and 
Table I. Description of the patient cohort.

A, Variables measured during the patients follow-up

\begin{tabular}{lcc}
\hline Variable & Mean \pm standard deviation & Range \\
\hline Age (years) & $68.3 \pm 13.1$ & $21.0-92.0$ \\
Time since diagnosis (years) & $6.5 \pm 5.8$ & $1.0-25.0$ \\
Body mass index & $24.4 \pm 4.6$ & $16.3-36.7$ \\
TKTL1 max & $150.83 \pm 44.90$ & $98.0-323.0$ \\
Apo10 max & $15.9 \pm 17.2$ & $9.0-66.6$ \\
Smoking habits (pack years) & $16.32 \pm 25.30$ & $0-120$ \\
Number of sessions & $4.02 \pm 2.80$ & $1.0-19.0$ \\
Duration between sessions (days) & $163.26 \pm 136.10$ & $31-684$ \\
\hline
\end{tabular}

$\mathrm{B}$, Characteristics of the patients

Characteristic $(n=78)$

Gender

Female

Male

Smoking habits (pack years)

0

1-20

Missing data

Tumour status

Type of cancer

Breast

Prostate

Colon

Melanoma

Lung

Other

Diet during the observation period

Not ketogenic

TKTL1, transketolase-like-1; TKTL1 max, maximum value of TKTL1 during the measurement period; Apo10 max, maximum value of the marker Apo10 during the measurement period.

Apo10, tumour stage and adherence to a ketogenic diet are displayed in Table III.

The distribution of TKTL1 values across the 3 groups of tumour stages is presented in Fig. 1. Significant differences were observed between these groups (Kruskal-Wallis, $\mathrm{P}=0.0002)$. Since the levels of TKTL1 depend on the activity of the immune system, which is often dysfunctional in late-stage cancer, the levels of TKTL1 were comparatively low in endstage patients, although the general trend was still visible. Fig. 2 presents the distribution of TKTL1 values according to the type of diet. Significant differences between the groups were observed (Kruskal-Wallis, $\mathrm{P}=0.0045$ ).

Of the 78 patients, $13(17 \%)$ had adopted a ketogenic diet, 6 of them partially (8\%) and 7 in full (9\%). As indicated in 
Table II. Number and percentage of patients with different stages of cancer that adopted a ketogenic diet.

\begin{tabular}{lcccc}
\hline Diet & Curative stage, $\mathrm{n}(\%)$ & Palliative stage, $\mathrm{n}(\%)$ & Endstage, $\mathrm{n}(\%)$ & Total, $\mathrm{n}(\%)$ \\
\hline Not ketogenic & $50(74)$ & $9(11)$ & $6(8)$ & $65(83)$ \\
Partially ketogenic & $3(4)$ & $2(2)$ & $1(1)$ & $6(8)$ \\
Fully ketogenic & $3(4)$ & $4(5)$ & $0(-)$ & $7(9)$ \\
Total & $56(72)$ & $15(19)$ & $7(9)$ & $78(100)$ \\
\hline
\end{tabular}

Table III. Nonparametric (Spearman's rank) correlations between TKTL1 max, Apo10 max, type of diet (no, partially or fully ketogenic) and the tumour stage (curative, palliative or endstage).

\begin{tabular}{lccc}
\hline Characteristics & TKTL1 max & Apo10 max & Ketogenic diet \\
\hline Tumour status & $0.47^{\mathrm{a}}$ & $0.32^{\mathrm{a}}$ & $0.26^{\mathrm{a}}$ \\
TKTL1 max & - & $0.34^{\mathrm{a}}$ & $0.33^{\mathrm{a}}$ \\
Apo10 max & - & - & 0.08 \\
\hline
\end{tabular}

TKTL1, transketolase-like-1; TKTL1 max, maximum value of TKTL1 during the observation period; Apo10 max, maximum value of Apo10 during the observation period; ${ }^{\mathrm{P}} \mathrm{P}<0.05$.

Table IV. Number of patients who modified their diet and experienced a variation in tumour status over time.

\begin{tabular}{lcccc}
\hline Diet & Improvement & No alteration & Worsening & Total \\
\hline Not ketogenic & 1 & 56 & 10 & 67 \\
Switch to partial ketogenic diet & 0 & 5 & 1 & 6 \\
Switch to full ketogenic diet & 3 & 2 & 0 & 5 \\
Total & 4 & 63 & 11 & 78 \\
\hline
\end{tabular}

Table II, none of the patients in endstage cancer adopted the diet fully, and 1 patient adhered partially to it. Of the 4 palliative patients that adopted a strict ketogenic diet, 1 patient with metastatic breast cancer, who had embraced a strict ketogenic diet since 2009, experienced a full remission of all metastases in the lung and bones. This was verified by fludeoxyglucose-PET analysis, which demonstrated no active metastases and 1 inactive metastasis in the thoracic region of the spine. In another palliative patient, who was diagnosed with cancer of the ovary in 1996 and had previously experienced cancer of the left and right breast in 1987 and 1998, respectively, followed by a recurrence of the carcinoma on the left side in 2006 and another recurrence in 2009, no further recurrences were detected during the observation period. A third patient with astrocytoma experienced a progression of the disease despite the ketogenic diet, although the high-dose corticosteroid therapy that the patient had received may have impacted their glucose metabolism. The fourth patient who adhered to a ketogenic diet did not undergo follow-up testing. Those patients who had started on a strict ketogenic diet, but then stopped it, experienced initially a remission of the disease or the metastases, which rebounded once they had stopped the diet, frequently with lethal consequences. A breakdown of the numbers of patients with modifications in diet by alteration in tumour status is displayed in Table IV. As indicated in the table, a correlation was observed between an improvement of the disease and having adopted the ketogenic diet in full $\left(\chi^{2}=33.26 ; \mathrm{df}=4\right.$; $\mathrm{P}=0.00001)$. Fig. 3 demonstrates that a reduction in TKTL1 score from baseline to the final measurement was associated with ketogenic diet and improvement in cancer status. It should be noted that, due to the insufficient number of cases available for statistical evaluation, this association is simply displayed for visual inspection.

Statistical analysis. In a logistic regression analysis, with endstage and palliative stage collapsed into 1 category, and where cancer stage was considered to be the dependent variable, whereas the value of TKTL1 and the adherence to a ketogenic diet were treated as the independent variables, the influence of these variables was estimated. The model was observed to be significant $\left(\chi^{2}, 22.7 ; \mathrm{P}=0.00001\right)$, with a parameter estimate for TKTL1 of $-0.034(95 \% \mathrm{CI}=-0.014$ to $-0.054 ; \mathrm{P}=0.001)$ and $\mathrm{OR}=0.96(95 \% \mathrm{CI}=0.95-0.99)$ for the unit variation, which implies that for every 0.034 unit reduction in TKTL1 values there is a $4 \%$ probability of improvement in tumour status, from palliative or lethal to curative stage. Other variables, including the duration of diagnosis or the maximum values of Apo10, were not significant predictors. 


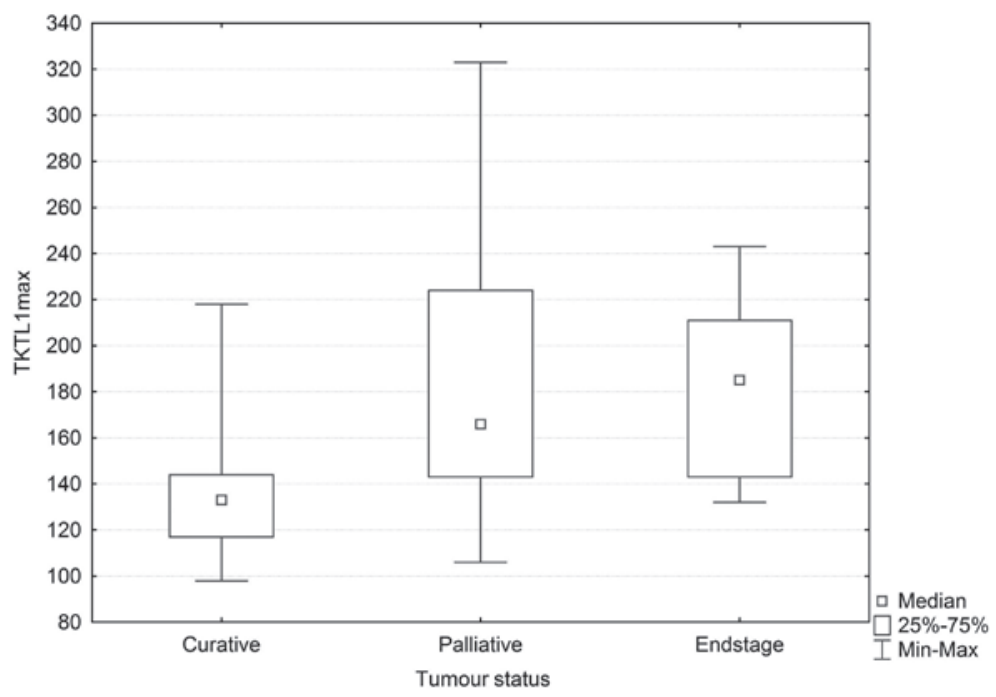

Figure 1. Maximum values of TKTL1 (TKTL1 max) according to tumour status. Box plot of the median, interquartile and full range by group. Significant differences were observed between the groups (Kruskal-Wallis test, $\mathrm{P}=0.0002$ ). TKTL1, transketolase-like-1; min, minimum; max, maximum.

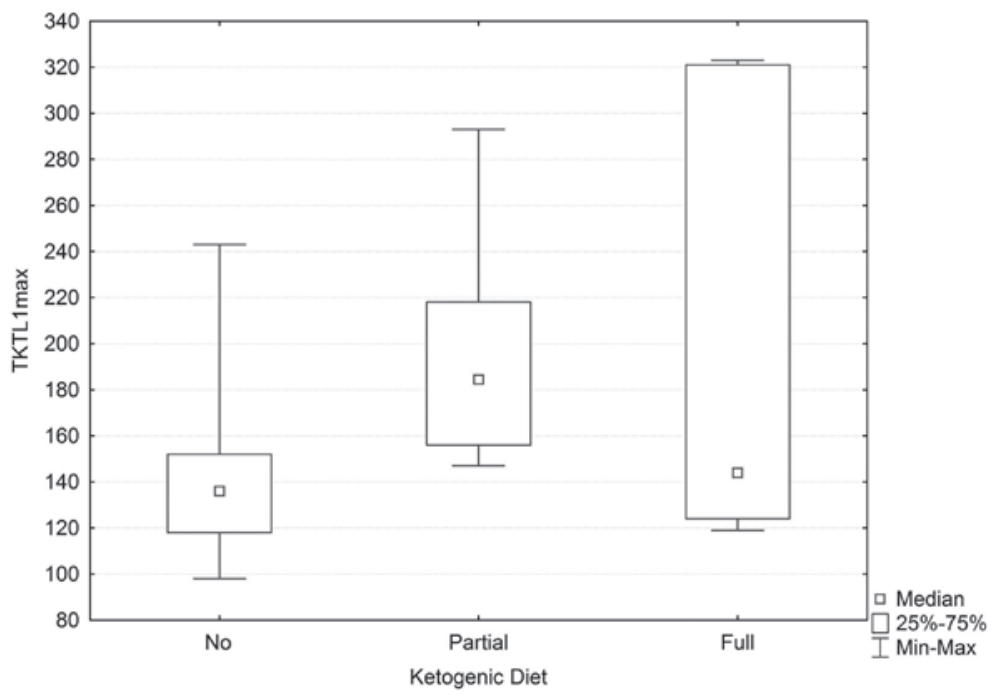

Figure 2. Maximum values of TKTL1 (TKTL1 max) according to diet. Box plot of the median, interquartile and full range by group. Significant differences were observed between the groups (Kruskal-Wallis test, $\mathrm{P}=0.0045$ ). TKTL1, transketolase-like-1; min, minimum; max, maximum.

\section{Discussion}

To the best of our knowledge, the present study is the first to describe the effects of a ketogenic diet in patients with cancer in primary care. All patients who presented in a primary care clinic with cancer were documented, and the adherence to a ketogenic diet and the variation in status of the tumour were monitored. The levels of TKTL1 were measured continuously in the patients. TKTL1 is a novel marker that is supposedly associated with tumour progression and switching of the tumour cells to aerobic glycolysis (49). The results from the present study support the following conclusions: i) TKTL1 is associated with a more active disease and a worse prognosis; ii) a ketogenic diet reduces the levels of TKTL1; and iii) increased levels of TKTL1 predict the development of endstage disease.

In view of the biochemical knowledge discussed in the present report, and the preliminary findings from previous studies, it can be proposed that patients with a diagnosis of cancer (except those with endstage disease) can adopt a ketogenic diet and benefit from it, since those patients who embraced a ketogenic diet in the present study were observed to experience a halt in the progression of the disease or complete remission.

Nonetheless, these results should be viewed with the appropriate caution, since they derive from a cohort of patients in primary care, which implies that, despite being treated to the best current oncological standards (which are high in Germany), patients who are disease-free often suffer from metastases or recurrences, as was the case in a number of patients in the present cohort. In addition, patients frequently want to know whether they can contribute to their health or boost their immune system. Thus, if they are informed that no alternative treatment is available other than waiting for a potential recurrence, patients are likely to consult with 


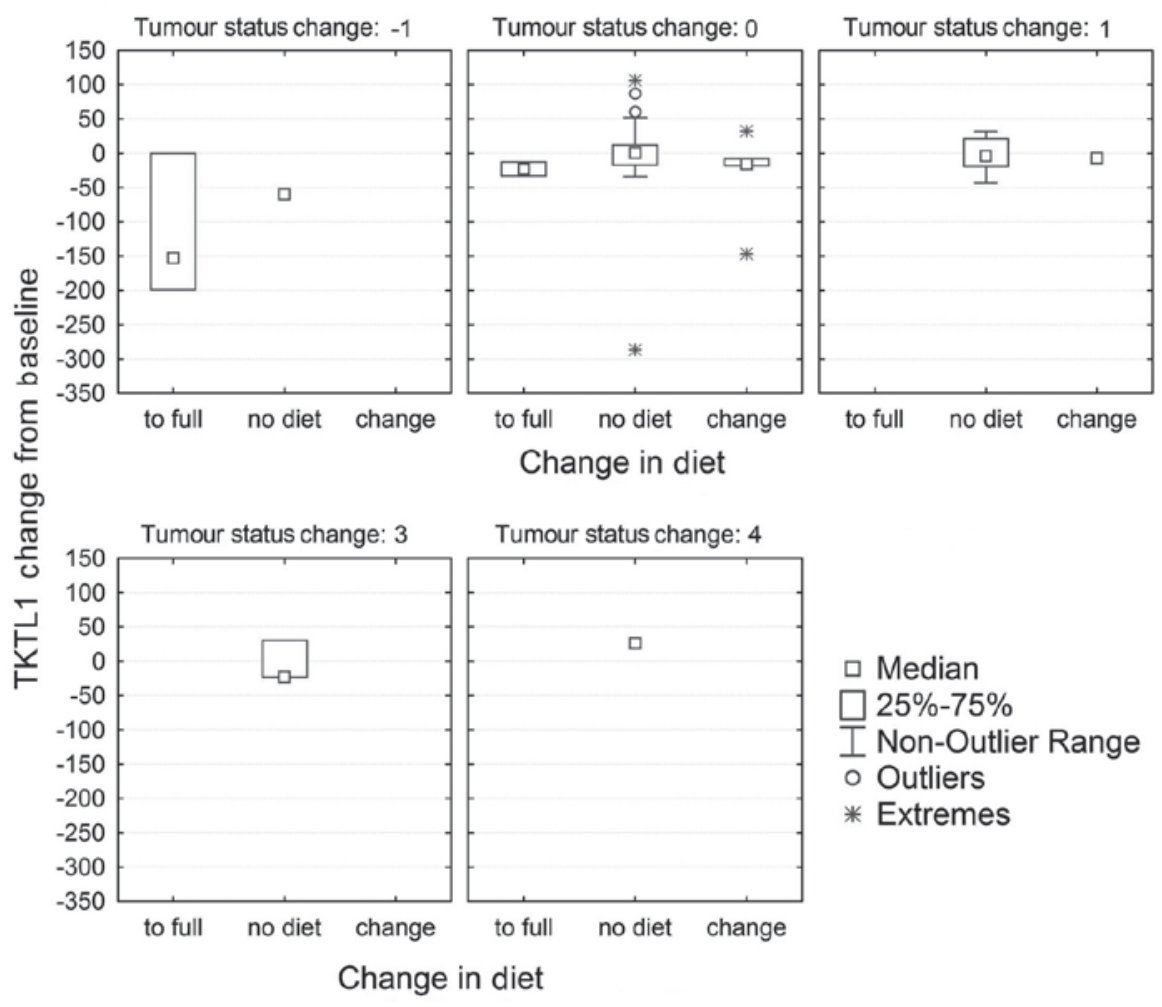

Figure 3. Change in TKTL1 scores from baseline according to ketogenic diet. Box plot of the variation in TKTL1 values grouped by adherence to a ketogenic diet (to full, complete adherence to a ketogenic diet; no diet, no adoption of ketogenic diet; or change, partial adherence to a ketogenic diet or a ketogenic diet was initiated but then discontinued), and categorised by the alteration observed in tumour status (-1, improvement; 0 , no modification/disease stable; $1-4$, worsening/disease progression). TKTL1, transketolase-like-1.

different practitioners (51). By contrast, offering the patients a diet which is comparatively natural and can be implemented by themselves strengthens their self-efficacy and expectancy of success, which, based on the current knowledge, may be an important variable in non-specific therapeutic effectiveness (52-56).

The present study was not a randomised study, but a study in general practice. The patients are likely to be representative of patients with cancer in the general population, since the clinic participates in the statutory German reimbursement system. However, since there is no control condition or control group matched for important predictors, it is difficult to preclude that the effects observed are not confounded. In fact, they possibly are, since patients with more progressed disease are less likely to adhere to the diet. Thus, the next important step would be to launch a large-scale randomised study to validate the effects of a ketogenic diet in the treatment of cancer.

In the present study, patients were offered the ketogenic diet that is provided by a company for ease of implementation, but this is not a requirement. Self-help books and brochures with recipes regarding ketogenic diet are available $(34,35)$, and certain patients may favour a more vegetarian type of ketogenic diet, which was not the focus of the current study $(4,57,58)$.

One of the aims of the present study was to investigate the effects of the novel marker TKTL1, which has been hypothesised to be a marker of switching of cells, particularly cancer cells, into a metabolic mode whereby they use glycolysis rather than oxidation for energy supply, in order to protect themselves from free radicals and to foster growth under anaerobic conditions such as those experienced by tumours growing at distant sites (36). While the present study was not intended to validate this hypothesis, the results obtained suggest that there is a positive link between the expression of TKTL1 and cancer progression, and a negative link between ketogenic diet and TKTL1 expression. This also explains why a ketogenic diet may be useful in patients with cancer, since cancer cells rarely use or are unable to use ketone bodies. Thus a ketogenic diet would withdraw the nutrients and energy requirements of a growing tumour, but not of the physiological functioning of the body. Therefore, reduced expression levels of TKTL1, which are a negative predictor of cancer progression, may reflect the effect of a ketogenic diet. To the best of our knowledge, the use of the EDIM test for detecting and measuring TKTL1 is not widespread. However, since the measurement is standardised, it may be useful to adopt the EDIM test more commonly as a predictor of disease processes where a ketogenic diet may be beneficial. In the present study, the correlation between ketogenic diet and TKTL1 was observed to be moderate in size and significant, explaining $22 \%$ of the common variance. However, it should be noted that in endstage disease, the marker loses its predictive value.

The positive correlation between ketogenic diet and tumour stage observed in the current study is likely to be artificial and due to the fact that more patients in a progressed stage of the disease were willing to adopt the diet. Naturally, they are also the patients who were most likely to benefit from it, as the data 
in Table IV and Fig. 3 suggest. The length of time that the diet needs to be maintained for is currently unknown. The present study covers an observation period of $\sim 2$ years, and a number of patients were able to follow the diet over the whole period. Clinical experience teaches that it is beneficial to maintain a ketogenic diet with active cancer $(59,60)$.

The results of the present study regarding the association between the modification of diet and the variation in tumour status must be considered with caution, since the number of observations for the majority of the variables reported is insufficient to perform a reliable statistical analysis, and hence, all the statistical estimations may be affected by error. Instead, they are intended to be taken as guidance and to encourage further research.

In summary, the present study has demonstrated that a ketogenic diet is feasible and likely beneficial for patients with cancer in primary care, who have been treated for their primary cancer, since it altered the expression of TKTL1, a novel and potentially useful marker to monitor the metabolic state of the body regarding aerobic glycolysis, and to evaluate the potential progression of non-endstage tumour disease. Therefore, the design of rigorous randomised studies to validate these observations is required.

\section{Acknowledgements}

The authors are grateful to Miss Majella Horan MSc, the native speaker editor who improved the style of the manuscript. The present study was a requirement of the $\mathrm{PhD}$ degree awarded to Dr Natalie Jansen, who holds shares in Tavarlin, a company that offers ketogenic products.

\section{References}

1. Sporn MB: The war on cancer. Lancet 347: 1377-1381, 1996.

2. Cancer in Germany $2009 / 2010,9^{\text {th }}$ edition. Contributions to the Health Reporting of the Federal Republic. Robert Koch Institut, Berlin, 2013 (In German).

3. Cancer. Fact Sheet 297. World Health Organisation, Geneva, 2014.

4. Paoli A, Rubini A, Volek JS and Grimaldi KA: Beyond weight loss: A review of the therapeutic uses of very-low-carbohydrate (ketogenic) diets. Eur J Clin Nutr 67: 789-796, 2013.

5. Ornish D, Weidner G, Fair WR, Marlin R, Pettengill EB, Raisin CJ, Dunn-Emke S, Crutchfield L, Jacobs FN and Barnard RJ: Intensive lifestyle changes may affect the progression of prostate cancer. J Urol 174: 1065-1070, 2005.

6. Berrino F, De Angelis R, Sant M, Rosso S, Bielska-Lasota M, Coebergh JW and Santaquilani M; Eurocare Working group: Survival for eight major cancers and all cancers combined for European adults diagnosed in 1995-99: Results of the EUROCARE-4 study. Lancet Oncol 8: 773-783, 2007.

7. De Angelis R, Sant M, Coleman MP, Francisci S, Baili P, Pierannunzio D, Trama A, Visser O, Brenner H, Ardanaz E, et al; EUROCARE-5 Working Group: Cancer survival in Europe 1999-2007 by country and age: Results of EUROCARE-5 - a population-based study. Lancet Oncol 15: 23-34, 2014.

8. Hölzel D and Engel J: We cannot neglect experiences from clinical practice. The example of metastasising colon tumour shows how clinical registers can help contribute to the evaluation of benefits of medications. Dtsch Arztebl 109: A424-A425, 2012 (In German).

9. Schlesinger-Raab A, Eckel R, Engel J, Sauer H, Löhrs U, Molls $\mathrm{M}$ and Hölzel D: Metastasising breast cancer: No prolongation of life since 20 years. Dtsch Arztebl 102: A2706-A2714, 2005 (In German).

10. Gray L, Lee IM, Sesso HD and Batty GD: Association of body mass index in early adulthood and middle age with future site-specific cancer mortality: The Harvard Alumni Health Study. Ann Oncol 23: 754-759, 2012.
11. Ford ES, Bergmann MM, Kröger J, Schienkiewitz A, Weikert C and Boeing H: Healthy living is the best revenge: Findings from the European Prospective Investigation Into Cancer and Nutrition - Potsdam study. Arch Intern Med 169: 1355-1362, 2009.

12. Gonzalez CA and Riboli E: Diet and cancer prevention: Contributions from the European Prospective Investigation into Cancer and Nutrition (EPIC) study. Eur J Cancer 46: 2555-2562, 2010.

13. Donaldson MS: Nutrition and cancer: A review of the evidence for an anti-cancer diet. Nutrit J 20: 19, 2004.

14. Bao Y, Han J, Hu FB, Giovannucci EL, Stampfer MJ, Willett WC and Fuchs CS: Association of nut consumption with total and cause-specific mortality. N Engl J Med 369: 2001-2011, 2013.

15. Ruskin DN and Masino SA: The nervous system and metabolic dysregulation: Emerging evidence converges on ketogenic diet therapy. Front Neurosci 6: 33, 2012.

16. Fontana L and Klein S: Aging, adiposity, and calorie restriction. JAMA 297: 986-994, 2007.

17. Henderson ST: High carbohydrate diets and Alzheimer's disease. Med Hypotheses 62: 689-700, 2004.

18. Eaton SB and Konner M: Paleolithic nutrition: A consideration of its nature and current implications. N Engl J Med 312: 283-289, 1985.

19. Mellberg C, Sandberg S, Ryberg M, Eriksson M, Brage S, Larsson C, Olsson T and Lindahl B: Long-term effects of a papaeolithic-type diet in obese postmenopausal women: A 2-year randomized trail. Eur J Clin Nutr 68: 350-357, 2014.

20. Warburg O, Posener K and Negelein E: On tumour metabolism. Biochem Z 152: 319-344, 1924 (In German).

21. Zhivotovsky B and Orrenius S: The Warburg Effect returns to the cancer stage. Semin Cancer Biol 19: 1-3, 2009 (Editorial).

22. Vander Heiden MG, Cantley LC and Thompson CB: Understanding the Warburg effect: The metabolic requirements of cell proliferation. Science 324: 1029-1033, 2009.

23. Garber K: Energy boost: The Warburg effect returns in a new theory of cancer. J Natl Cancer Inst 96: 1805-1806, 2004.

24. Romano AH and Conway T: Evolution of carbohydrate metabolic pathways. Res Microbiol 147: 448-455, 1996.

25. Newington JT, Pitts A, Chien A, Arseneault R, Schubert D and Cumming RC: Amyloid $\beta$ resistance in nerve cell lines is mediated by the Warburg effect. PLoS One 6: e19191, 2011.

26. Gogvadze V, Zhivotovsky B and Orrenius S: The Warburg effect and mitochondrial stability in cancer cells. Mol Aspects Med 31: 60-74, 2010.

27. Weinhouse S: The Warburg hypothesis fifty years later. Z Krebsforsch Klin Onkol 87: 115-126, 1976.

28. Holm E and Kämmerer U: Fat and carbohydrates in diets for tumour patients. Aktuel Ernahrungsmed 36: 286-298, 2011 (In German).

29. Klement RJ and Kämmerer U: Is there a role for carbohydrate restriction in the treatment and prevention of cancer? Nutr Metab (Lond) 8: 75, 2011.

30. Otto C, Klingelhöffer C, Biggemann L, Melkus G, Mörchel P, Jürgens C, Gahn S and Kämmerer U: Experimental studies on the metabolism of keton bodies and lactate by tumour cells of the gastrointestinal duct. Aktuel Ernahrungsmed 39: 51-59, 2014 (In German)

31. Vaughn AE and Deshmukh M: Glucose metabolism inhibits apoptosis in neurons and cancer cells by redox inactivation of cytochrome c. Nat Cell Biol 10: 1477-1483, 2008.

32. Gunter MJ, Hoover DR, Yu H, Wassertheil-Smoller S, Rohan TE, Manson JE, Li J, Ho GY, Xue X, Anderson GL, et al: Insulin, insulin-like growth factor-I, and risk of breast cancer in postmenopausal women. J Natl Cancer Inst 101: 48-60, 2009.

33. Schmidt M, Pfetzer N, Schwab M, Strauss I and Kämmerer U: Effects of a ketogenic diet on the quality of life in 16 patients with advanced cancer: A pilot trial. Nutr Metab (Lond) 8: 54, 2011.

34. Kämmerer U: Ketogenic diet for cancer patients. The University Hospital Würzburg, Würzburg, Germany, 2010 (In German).

35. Coy JF and Franz M: The new anti-cancer diet: How you can stop the cancer gene. Volume 1. 1st edition. Gräfe und Unzer, Munich, 2009.

36. Coy JF, Dressler D, Wilde J and Schubert P: Mutations in the transketolase-like gene TKTL1: Clinical implications for neurodegenerative diseases, diabetes and cancer. Clin Lab 51: 257-273, 2005.

37. Langbein S, Zerilli M, Zur Hausen A, Staiger W, Rensch-Boschert K, Lukan N, Popa J, Ternullo MP, Steidler A, Weiss C, et al: Expression of transketolase TKTL1 predicts colon and urothelial cancer patient survival: Warburg effect reinterpreted. Br J Cancer 94: 578-585, 2006. 
38. Sun W, Liu Y, Glazer CA, Shao C, Bhan S, Demokan S, Zhao M, Rudek MA, Ha PK and Califano JA: TKTL1 is activated by promoter hypomethylation and contributes to head and neck squamous cell carcinoma carcinogenesis through increased aerobic glycolysis and HIFl $\alpha$ stabilization. Clin Cancer Res 16: 857-866, 2010.

39. Schwaab J, Horisberger K, Ströbel P, Bohn B, Gencer D, Kähler G, Kienle P, Post S, Wenz F, Hofmann WK, et al: Expression of Transketolase like gene 1 (TKTL1) predicts disease-free survival in patients with locally advanced rectal cancer receiving neoadjuvant chemoradiotherapy. BMC Cancer 11: 363, 2011.

40. Zhang S, Yang JH, Guo CK and Cai PC: Gene silencing of TKTL1 by RNAi inhibits cell proliferation in human hepatoma cells. Cancer Lett 253: 108-114, 2007.

41. Grimm M, Schmitt S, Teriete P, Biegner T, Stenzl A, Hennenlotter J, Muhs HJ, Munz A, Nadtotschi T, König K, et al: A biomarker based detection and characterization of carcinomas exploiting two fundamental biophysical mechanisms in mammalian cells. BMC Cancer 13: 569, 2013.

42. Kayser G, Sienel W, Kubitz B, Mattern D, Stickeler E, Passlick B, Werner $\mathrm{M}$ and Zur Hausen A: Poor outcome in primary non-small cell lung cancers is predicted by transketolase TKTL1 expression. Pathology 43: 719-724, 2011.

43. Lange C, Tisch-Rottensteiner J, Böhringer D, Martin G, Schwartzkopff J and Auw-Haedrich C: Enhanced TKTL1 expression in malignant tumors of the ocular adnexa predicts clinical outcome. Ophthalmology 119: 1924-1929, 2012.

44. Feyen O, Coy JF, Prasad V, Schierl R, Saenger J and Baum RP EDIM-TKTL 1 blood test: A noninvasive method to detect upregulated glucose metabolism in patients with malignancies. Future Oncol 8: 1349-1359, 2012.

45. Jansen N and Coy JF: Diagnostic use of epitope detection in monocytes blood test for early detection of colon cancer metastasis. Future Oncol 9: 605-609, 2013.

46. Mayer A, Von Wallbrunn A and Vaupel P: Glucose metabolism of malignant cells is not regulated by transketolase-like (TKTL)-1. Int J Oncol 37: 265-271, 2010.

47. Rotmann AR, Hofmann HA and Coy JF: Apo10 - a new biomarker for early detection of disorders of cell proliferation and solid tumours. Abstracts of XX FIGO World Congress of Gynecology and Obstetrics. Int J Gynecol Obstet 119 (Suppl 3): S466, 2012.

48. Fine EJ, Segal-Isaacson CJ, Feinman RD, Herszkopf S, Romano MC, Tomuta N, Bontempo AF, Negassa A and Sparano JA: Targeting insulin inhibition as a metabolic therapy in advanced cancer: A pilot safety and feasibility dietary trial in 10 patients. Nutrition 28: 1028-1035, 2012.
49. Wanka C, Steinbach JP and Rieger J: Tp53-induced glycolysis and apoptosis regulator (TIGAR) protects glioma cells from starvation-induced cell death by up-regulating respiration an dimproving cellular redox homeostasis. J Biol Chem 287: 33436-33446, 2012.

50. Rieger J, Bähr O, Maurer GD, Hattingen E, Franz K, Brucker D, Walenta S, Kämmerer U, Coy JF, Weller M and Steinbach JP: ERGO: A pilot study of ketogenic diet in recurrent glioblastoma. Int J Oncol 44: 1843-1852, 2014.

51. Guethlin C, Walach H, Naumann J, Bartsch HH and Rostock M: Characteristics of cancer patients using homeopathy compared with those in conventional care: A cross-sectional study. Ann Oncol 21: 1094-1099, 2010.

52. Segar J: Complementary and alternative medicine: Exploring the gap between evidence and usage. Health Lond 16: 366-381, 2012.

53. Walach H: Spiritual and alternative cancer therapies: Strange theories, perhaps wrong, but useful. Breast Care (Basel) 2: 190-192, 2007.

54. Chvetzoff G and Tannock IF: Placebo effects in oncology. J Natl Cancer Inst 95: 19-29, 2003.

55. Walach $\mathrm{H}$ (ed): Placebo effects in Complementary and Alternative Medicine: The selfhealing response. In Placebo and Pain: From Bench to Bedside. Elsevier-Academic Press, Amsterdam, pp189-202, 2013.

56. Walach H: Placebo controls: Historical, methodological and general aspects. Philos Trans R Soc London B Biol Sci 366: 1870-1878, 2011

57. Fontana L, Partridge L and Longo VD: Extending healthy life span - from yeast to humans. Science 328: 321-326,2010.

58. Mutter J: Healthy instead of chronically ill! The holistic path: prevention instead of treatment. Fit fürs Leben Verlag, Weil der Stadt, 2009 (In German)

59. Ho V, Leung K, Hsu A, Luk B, Lai Jm Shen SY, Minchinton AI, Waterhouse D, Bally MB, Lin W, et al: A low carbohydrate, high protein diet slows tumor growth and prevents cancer initiation. Cancer Res 71; 4484-4492, 2011.

60. Masko EM, Thomas JA, Antonelli JA, Lloyd JC, Phillips TE, Poulton SH, Dewhirst MW, Pizzo SV, Freedland SJ: Low-carbohydrate diets anmd prostate cancer: How low is 'low enough'?: Cancer Prev Res (Phila) 3: 1124-1131, 2010. 